\title{
Icatibant for Multiple Hereditary Angioedema Attacks across the Controlled and Open-Label Extension Phases of FAST-3
}

\author{
William R. Lumry ${ }^{a}$ Henriette Farkas ${ }^{b}$ Dumitru Moldovan ${ }^{c}$ Elias Toubi ${ }^{d}$ \\ Jovanna Baptista ${ }^{e}$ Timothy Craig $^{f}$ Marc Riedlg \\ ${ }^{a}$ AARA Research Center, Dallas, Tex., USA; ${ }^{b}$ Third Department of Internal Medicine, Semmelweis University, \\ Budapest, Hungary; ' Allergology-Immunology Department, Mures County Hospital, University of Medicine and \\ Pharmacy, Tirgu-Mures, Romania; ${ }^{d}$ Division of Allergy and Immunology, Bnai Zion Medical Center, Haifa, Israel;

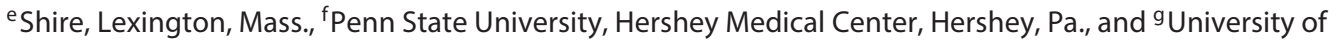 \\ California - San Diego, La Jolla, Calif., USA
}

\section{Key Words}

Abdominal - Acute treatment - C1-inhibitor deficiency .

Cutaneous - FAST-3 - Hereditary angioedema - Icatibant .

Laryngeal $\cdot$ Open-label extension

\begin{abstract}
Background: In randomized, controlled, double-blind, multicenter phase 3 studies, one icatibant injection was efficacious and generally well tolerated in patients with a single hereditary angioedema (HAE) attack. Here, the efficacy and safety of icatibant for multiple HAE attacks was evaluated across the controlled and open-label extension phases of the For Angioedema Subcutaneous Treatment (FAST)-3 study (NCT00912093). Methods: In the controlled phase, adults with HAE type I or II were randomized (1:1) to receive a single subcutaneous injection of icatibant $30 \mathrm{mg}$ or placebo within $6 \mathrm{~h}$ of an attack becoming mild (laryngeal) or moderate (cutaneous/abdominal). Open-label icatibant was administered for severe laryngeal symptoms. In the openlabel extension phase, patients could receive up to three icatibant injections per attack. Efficacy and safety were analyzed for the first five icatibant-treated attacks at any location (prospective analysis) and laryngeal attacks (post hoc
\end{abstract}

analysis) across both phases. Efficacy outcomes were based on patient-reported symptom severity (visual analog scale). Results: In groups of patients with one to five icatibant-treated attacks at any location $(n=88)$, the median times to onset of symptom relief, onset of primary symptom relief and almost complete symptom relief were 1.9-2.1, 1.5-2.0 and 3.5-19.7 $\mathrm{h}$, respectively. The same outcomes for laryngeal attacks $(n=25)$ were 1.0-2.0, 1.0-2.0 and 1.5-8.1 h, respectively. The most frequently reported adverse events were a worsening or recurrence of HAE attack, headache and nasopharyngitis. Two serious adverse events (arrhythmia and noncardiac chest pain) were considered to be related to icatibant. Conclusions: Icatibant was efficacious and generally well tolerated across multiple HAE attacks, including laryngeal attacks.

(c) 2015 S. Karger AG, Basel

\section{Introduction}

Hereditary angioedema (HAE) is a rare autosomal dominant disease characterized by recurrent and unpredictable episodes of localized swelling of the skin, gastrointestinal tract and/or upper respiratory tract [1]. In the

\section{KARGER 125}

2015 S. Karger AG, Base

$1018-2438 / 15 / 1681-0044 \$ 39.50 / 0$
Correspondence to: Dr. William R. Lumry

AARA Research Center

Dallas, TX 75231 (USA)

E-Mail LumryMD@AllergySpecialists.us 
most serious cases, laryngeal edema results in upper airway obstruction and can lead to death by asphyxiation if not treated promptly $[2,3]$. The edema is mediated by elevated bradykinin levels associated with a deficiency (type I) or functional loss (type II) of C1-esterase inhibitor $[4,5]$. HAE with normal C1-esterase inhibitor has more recently been described [6], but was not a subject of the present study.

Icatibant is a bradykinin B2 receptor antagonist that demonstrated efficacy compared with placebo, as well as a favorable safety profile, when used for the treatment of edematous attacks in patients with HAE type I or II in the randomized, controlled, double-blind phases of the For Angioedema Subcutaneous Treatment (FAST)-1, FAST2 and FAST-3 phase 3 studies (ClinicalTrials.gov identifiers: NCT00097695, NCT00500656 and NCT00912093, respectively; see Cicardi et al. [7] and Lumry et al. [8] for further details). The patients enrolled in these studies had experienced numerous HAE attacks in the 6 months prior to baseline, highlighting that patients frequently require treatment for multiple HAE attacks over time. With this in mind, the use of icatibant for the acute treatment of multiple HAE attacks was evaluated in the open-label extension (OLE) phases of each of the phase 3 studies. The results of the FAST-1 and FAST-2 OLE phases have previously been published: both studies reported that the efficacy and safety profiles of icatibant remained consistent over the treatment of multiple attacks $[9,10]$. Here, we report data from the controlled and OLE phases of FAST-3 to further evaluate the efficacy and safety of icatibant for the repeated treatment of multiple HAE attacks at any location, and also specifically for multiple laryngeal attacks.

\section{Material and Methods}

\section{Study Design}

FAST-3 was a randomized, controlled, double-blinded, multicenter phase 3 study conducted at 67 centers in 11 countries. The controlled phase enrolled 98 eligible patients, $\geq 18$ years of age, diagnosed with HAE type I or type II who were experiencing a moderate to very severe cutaneous or abdominal HAE attack, or a mild to moderate laryngeal HAE attack (full inclusion and exclusion criteria have been reported previously [8]).

The study was conducted in compliance with the US Food and Drug Administration Institutional Review Board regulations, the good clinical practice guidelines of the International Conference on Harmonization, and the Declaration of Helsinki. The protocol, protocol amendments and patient informed consent forms were reviewed and approved by the institutional review board at each participating institution. All patients gave written informed consent.

Icatibant for Multiple HAE Attacks in FAST-3

\section{Treatment}

In the controlled phase of the study, patients with eligible HAE attacks were randomized $(1: 1)$ to receive a single subcutaneous (s.c.) injection of icatibant $30 \mathrm{mg}$ or placebo (an isotonic, sterile and buffered solution for s.c. injection). Icatibant or placebo was administered to the patient by the investigator or authorized health professional at the study site. Patients were to receive study treatment within $12 \mathrm{~h}$ of the onset of their first attack, and within $6 \mathrm{~h}$ of the attack becoming at least mild (laryngeal) or moderate (cutaneous and/or abdominal) in severity (investigator global assessment based on a 5-point scale from 0 for 'absence of symptoms' to 4 for 'very severe symptoms'). Patients with severe laryngeal symptoms were not randomized but could receive open-label treatment with icatibant $30 \mathrm{mg}$ s.c.

After the first attack, all patients were eligible to enter an OLE phase, in which open-label icatibant $30 \mathrm{mg}$ s.c. was administered for the treatment of any subsequent HAE attacks that were severe enough to warrant treatment. A maximum of three injections $6 \mathrm{~h}$ apart were permitted if the attack worsened or if, in the opinion of the investigator, symptoms remained severe enough to warrant further treatment. If symptoms worsened more than $48 \mathrm{~h}$ after the initial treatment, the event was to be considered a new attack.

Other acute treatments for HAE attacks were permitted as rescue therapies (e.g. morphine sulfate or an equivalent low-dose narcotic, antiemetics, fresh frozen plasma, epinephrine, C1-inhibitor concentrate and nonsteroidal anti-inflammatory drugs). For the purposes of the trial, rescue therapy was defined as any medication that, in the opinion of the investigator, was immediately necessary to alleviate acute symptoms resulting from the current HAE attack.

\section{Assessments}

Pretreatment assessments were performed prior to randomization and subsequent treatment. Patients assessed symptoms using a $100 \mathrm{~mm}$ visual analog scale (VAS; higher scores indicated increased severity; one VAS for each symptom) at pretreatment, every $30 \mathrm{~min}$ from 1 to $4 \mathrm{~h}$ after treatment, at 5, 6, 8 and $12 \mathrm{~h}$ after treatment, and then three times daily on days 2 and 3 , and once daily on days 4 and 5 . Three symptoms were assessed for cutaneous/abdominal attacks (skin swelling, skin pain and abdominal pain) and five symptoms were assessed for laryngeal attacks (skin swelling, skin pain, abdominal pain, difficulty swallowing and voice change). Attacks may have involved multiple locations; therefore, cutaneous/abdominal symptoms were reported in some patients with laryngeal attacks. The mean of the individual VAS scores for these symptoms was used to derive a composite VAS score.

The primary efficacy end point was the time to onset of symptom relief, defined as the earliest of three consecutive time points at which at least a $50 \%$ reduction in the patient-reported composite VAS score was achieved. The key secondary efficacy end point was the time to onset of primary symptom relief. For abdominal attacks, the primary symptom was abdominal pain; for cutaneous attacks, the primary symptom was either skin swelling or skin pain, whichever was more severe at pretreatment (if both were equally severe, then skin pain was used as the primary symptom). For laryngeal attacks, the primary symptom was either difficulty swallowing or voice change, whichever was more severe at pretreatment (if both were equally severe, then difficulty swallowing was used as the primary symptom). The onset of primary symptom relief was defined as the earliest of three consecutive time points at which the VAS score for the primary symptom was below the line $\mathrm{Y}=6 / 7 \times \mathrm{X}-16$, with $\mathrm{X} \geq 30 \mathrm{~mm}$ (where 
Fig. 1. Patient disposition. ${ }^{1}$ At the time of the previously reported interim analysis of the controlled phase of FAST-3 (see Lumry et al. [8]), there were 369 screened patients who had provided informed consent; here final study data from a later time point included 1 additional patient who provided informed consent, leading to a final population of $370 .{ }^{2}$ One patient with a cutaneous attack did not receive randomized treatment with placebo and was instead treated with open-label icatibant due to the development of severe laryngeal symptoms; this attack was considered an icatibant-treated laryngeal attack. Another patient with a cutaneous attack received randomized treatment with placebo, but was later treated with icatibant as a rescue medication; this attack was considered a placebo-treated cutaneous attack. ${ }^{3}$ One patient with a moderate laryngeal attack received randomized treatment with placebo, but was later treated with icatibant as a rescue medication; this attack was considered a placebo-treated laryngeal attack.

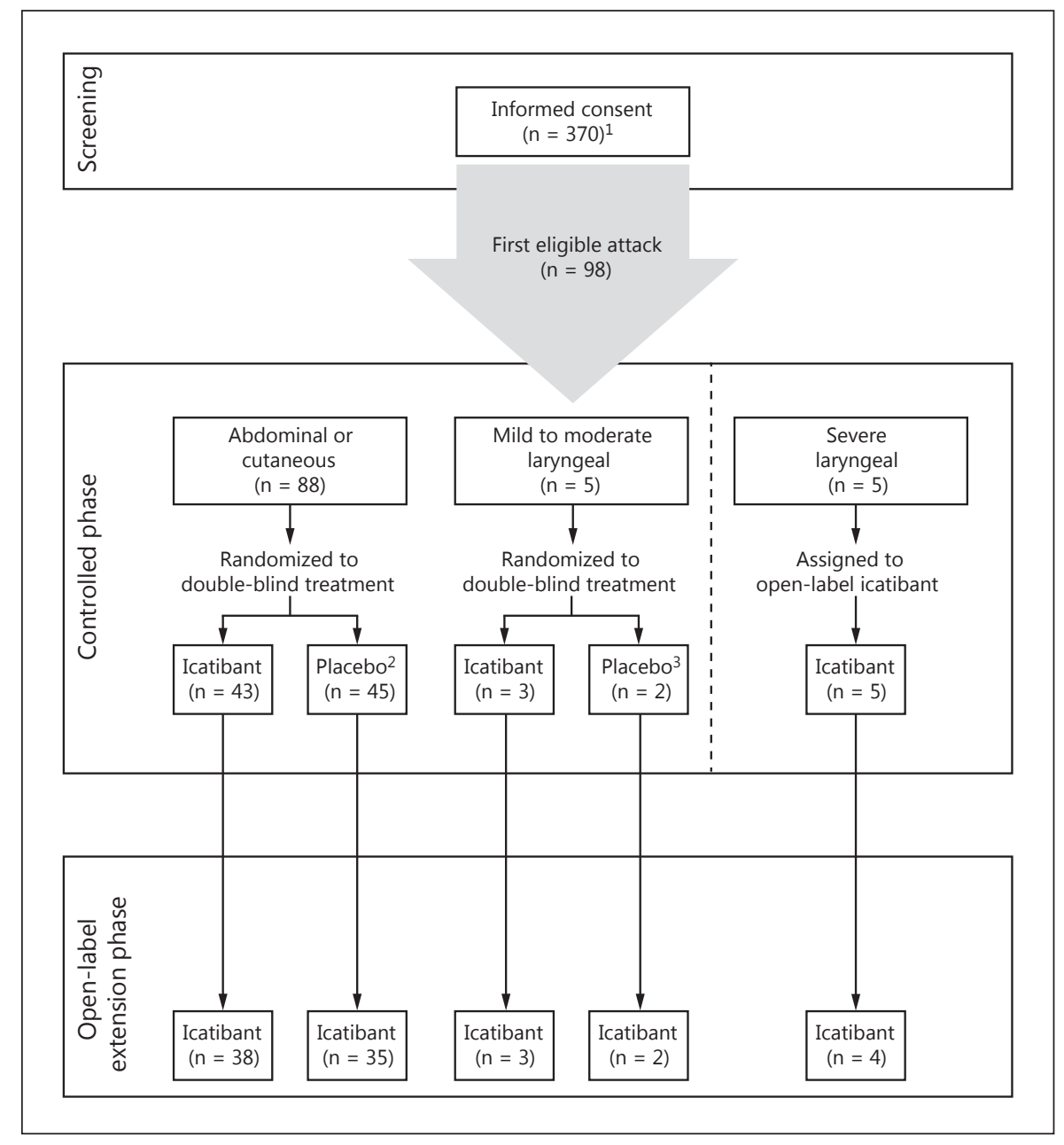

$\mathrm{X}=$ pretreatment VAS and $\mathrm{Y}=$ posttreatment VAS). This criterion corresponded to a reduction of $31 \mathrm{~mm}$ from a pretreatment VAS of $100 \mathrm{~mm}$, and $21 \mathrm{~mm}$ from a pretreatment VAS of $30 \mathrm{~mm}$. If the pretreatment VAS was $<30 \mathrm{~mm}$, primary symptom relief was defined as a $68 \%$ reduction from the pretreatment VAS score.

Other secondary end points included: the time to almost complete symptom relief, defined as the earliest of three consecutive time points at which all individual VAS scores were $<10 \mathrm{~mm}$; the time to initial symptom improvement, defined by the patient or investigator as the time that symptoms started to improve; change from pretreatment in composite VAS score; and changes from pretreatment in individual VAS symptom scores. All adverse events (AEs) were recorded, including those that may have represented clinically relevant worsening of the signs and symptoms of a treated HAE attack. Local tolerability at the injection site was documented separately from AEs.

\section{Statistical Analyses}

In this exploratory analysis of the FAST-3 data, the efficacy of repeated treatment with icatibant was evaluated prospectively for each of the first five icatibant-treated HAE attacks across the controlled and OLE phases, regardless of location (repeated-treatment population). Although six or more HAE attacks could still be treat- ed with icatibant, an initial analysis indicated insufficient numbers for inclusion, and therefore the final analysis reported here was limited to the first five icatibant-treated HAE attacks only. Efficacy was also evaluated post hoc for each of the first five icatibant-treated laryngeal attacks across the controlled and OLE phases (laryngeal repeated-treatment population).

For both the prospective analysis of treatment across multiple attacks, regardless of location, and the post hoc analysis across multiple laryngeal attacks, Kaplan-Meier methods were used to estimate the proportion of patients achieving each end point (onset of symptom relief, onset of primary symptom relief, almost complete symptom relief and initial symptom improvement) at a given time, and the median times and corresponding 95\% confidence intervals (CI). In addition, VAS scores were summarized using descriptive statistics at pretreatment and 1 and $4 \mathrm{~h}$ posttreatment. It should be noted that this was an exploratory analysis, which was not powered to draw statistical comparisons between treatment effects across the different icatibant-treated attacks.

AE tabular summaries were based on all treatment-emergent AEs occurring within 16 days of treatment administration. AEs occurring more than 16 days after treatment administration were captured and recorded on the case report form, but were excluded from the analyses. 
Table 1. Baseline patient characteristics (repeated-treatment population)

\begin{tabular}{|c|c|c|c|c|c|}
\hline Mean age, years (SD) & $37.1(12.6)$ & $37.1(13.3)$ & $36.7(13.4)$ & $39.6(13.6)$ & $39.7(14.1)$ \\
\hline \multicolumn{6}{|l|}{ Race, n (\%) } \\
\hline Caucasian & $78(88.6)$ & $62(88.6)$ & $47(85.5)$ & $31(83.8)$ & $25(80.6)$ \\
\hline \multicolumn{6}{|l|}{ HAE type, n (\%) } \\
\hline I & $77(87.5)$ & $62(88.6)$ & $49(89.1)$ & $33(89.2)$ & $28(90.3)$ \\
\hline II & $11(12.5)$ & $8(11.4)$ & $6(10.9)$ & $4(10.8)$ & $3(9.7)$ \\
\hline Median number of cutaneous attacks in the previous 6 months $^{1}$ (range) & $3.0(0-60)$ & $3.0(0-48)$ & $3.0(0-48)$ & $3.0(0-48)$ & $4.0(0-48)$ \\
\hline Median number of abdominal attacks in the previous 6 months ${ }^{2}$ (range) & $2.0(0-25)$ & $2.5(0-25)$ & $3.0(0-25)$ & $2.5(0-25)$ & $2.5(0-25)$ \\
\hline Median number of laryngeal attacks in the previous 6 months $^{3}$ (range) & $0.0(0-12)$ & $0.0(0-12)$ & $0.0(0-12)$ & $0.0(0-12)$ & $0.0(0-12)$ \\
\hline
\end{tabular}

${ }^{1}$ Data available for the following patient numbers: first attack, $\mathrm{n}=87$; second attack, $\mathrm{n}=70$; third attack, $\mathrm{n}=55$; fourth attack, $\mathrm{n}=37$; fifth attack, $\mathrm{n}=31$.

${ }^{2}$ Data available for the following patient numbers: first attack, $n=86$; second attack, $n=68$; third attack, $n=53$; fourth attack, $n=36$; fifth attack, $n=30$.

${ }^{3}$ Data available for the following patient numbers: first attack, $n=79$; second attack, $n=62$; third attack, $n=47 ;$ fourth attack, $n=32 ;$ fifth attack, $n=27$.

Table 2. HAE attack characteristics (repeated-treatment population)

\begin{tabular}{|c|c|c|c|c|c|}
\hline Characteristic & \multicolumn{5}{|c|}{ Icatibant-treated attack } \\
\hline Cutaneous & $43(48.9)$ & $28(40.0)$ & $24(43.6)$ & $18(48.6)$ & $15(48.4)$ \\
\hline Abdominal & $33(37.5)$ & $32(45.7)$ & $24(43.6)$ & $16(43.2)$ & $11(35.5)$ \\
\hline Laryngeal & $11(12.5)$ & $10(14.3)$ & $7(12.7)$ & $3(8.1)$ & $5(16.1)$ \\
\hline 1 & $85(96.6)$ & $70(100.0)$ & $51(92.7)$ & $36(97.3)$ & $31(100.0)$ \\
\hline 2 & $2(2.3)$ & $0(0.0)$ & $4(7.3)$ & $1(2.7)$ & $0(0.0)$ \\
\hline 3 & $1(1.1)$ & $0(0.0)$ & $0(0.0)$ & $0(0.0)$ & $0(0.0)$ \\
\hline
\end{tabular}

${ }^{1}$ Location of primary symptom.

${ }^{2}$ More than one injection of icatibant was used to treat: 1 patient with a cutaneous attack (fourth icatibant-treated attack), 5 patients with abdominal attacks [first $(n=2)$ or third $(n=3)$ icatibant-treated attack], 1 patient with a laryngeal attack (third icatibant-treated attack), and 1 patient with an attack at an unknown location (first icatibant-treated attack).

\section{Results}

\section{Patients}

The first patient was enrolled in FAST-3 in July 2009, with the last patient follow-up visit in July 2012. Of 98 enrolled patients, 93 were randomized to receive either icatibant $(n=46)$ or placebo $(n=47)$ in the controlled phase, 5 received open-label icatibant in the controlled phase (for severe laryngeal attacks), and 82 received openlabel icatibant in the OLE phase (fig. 1).

Icatibant for Multiple HAE Attacks in FAST-3
Prospective Analysis of Icatibant for Multiple Attacks, Regardless of Location

Of the 98 enrolled patients, 88 had at least one icatibant-treated HAE attack in the controlled and/or OLE phases and were included in the repeated-treatment population (the 10 other patients did not receive icatibant because they were randomized to placebo in the controlled phase and did not enter the OLE phase). When these patients were grouped according to the number of icatibant-treated HAE attacks (one to five), the patient 
characteristics were broadly consistent, with a mean age of 36.7-39.7 years (table 1).

For the first, second, third, fourth or fifth icatibanttreated HAE attacks, the majority of attacks were cutaneous (40.0-48.9\%) or abdominal (35.5-45.7\%), and the majority (92.7-100.0\%) were treated with a single injection (in the OLE phase, patients could receive a maximum of three injections; table 2). The first, second, third, fourth or fifth icatibant-treated HAE attacks in this population accounted for 281 of 487 icatibant-treated attacks (57.7\%) experienced during the entire FAST-3 study (the remaining attacks were the sixth or later icatibant-treated attacks).

Across the groups of patients with first, second, third, fourth or fifth icatibant-treated HAE attacks, the median time to onset of symptom relief was 1.9-2.1 h (fig. 2a). The median time to onset of primary symptom relief was 1.5-2.0 h (fig. 2b), the median time to almost complete symptom relief was 3.5-19.7 h (fig. 2c), and the median time to initial symptom improvement was $0.5-0.8 \mathrm{~h}$ when assessed by the patient, and $0.6-0.9 \mathrm{~h}$ when assessed by the investigator (fig. 2d, e, respectively). Largely overlapping 95\% CI supported the consistency of each of these outcomes across the multiple icatibant-treated attacks. Figure 3 shows the composite VAS scores and individual VAS symptom scores before treatment and at 1 and $4 \mathrm{~h}$ after treatment.

\section{Post hoc Analysis of Multiple Laryngeal Attacks}

Twenty-seven patients experienced a total of 51 icatibant-treated laryngeal attacks (laryngeal repeated-treatment population): first attack, $\mathrm{n}=27$; second, $\mathrm{n}=12$; third, $\mathrm{n}=5$; fourth, $\mathrm{n}=4$, and fifth, $\mathrm{n}=3$. The majority of these attacks $(49 / 51 ; 96.1 \%)$ were treated with a single injection; two icatibant-treated laryngeal attacks in 2 different patients required two injections (second icatibanttreated attack, $\mathrm{n}=1$; third icatibant-treated attack, $\mathrm{n}=1$ ) and none required three injections.

Across patients grouped by icatibant-treated laryngeal attack number (one to five), the median time to onset of symptom relief was 1.0-2.0 h (interquartile range, IQR, 1st attack: $1.3-3.5 \mathrm{~h} ; 2$ nd: $1.0-2.3 \mathrm{~h} ; 3$ rd: $1.0-1.5 \mathrm{~h} ; 4$ th: $1.0-1.7 \mathrm{~h} ; 5$ th: $1.0-1.0 \mathrm{~h}$; fig. $4 \mathrm{a}$ ), the median time to onset of primary symptom relief was $1.0-2.0 \mathrm{~h}$ (IQR 1 st attack: 1.3-2.5 h; 2 nd: $1.0-2.3 \mathrm{~h}$; $3 \mathrm{rd}: 1.0-1.5 \mathrm{~h} ; 4$ th: $1.0-1.5 \mathrm{~h}$; 5th: $1.0-1.0 \mathrm{~h}$; fig. $4 \mathrm{~b}$ ), and the median time to almost complete symptom relief was $1.5-8.1 \mathrm{~h}$ (IQR 1st attack: 2.2-25.5 h; 2nd: $2.5-25.4$ h; 3rd: 3.4-20.2 h; 4th: 1.5-3.7 h; 5 th: $1.0-1.5 \mathrm{~h}$; fig. $4 \mathrm{c}$ ). The median time to initial symptom improvement was consistently $<1 \mathrm{~h}$, whether assessed by the patient (IQR 1st attack: $0.3-1.6 \mathrm{~h} ; 2$ nd: $0.4-$
$1.5 \mathrm{~h}$; 3rd: $0.2-0.3 \mathrm{~h}$; 4 th: $0.3-0.4 \mathrm{~h}$; 5 th: $0.0-0.7 \mathrm{~h}$ ) or investigator (IQR 1st attack: $0.4-1.5 \mathrm{~h} ; 2$ nd: $0.5-1.5 \mathrm{~h} ; 3 \mathrm{rd}$ : $0.3-0.5 \mathrm{~h} ; 4$ th: $0.3-0.4 \mathrm{~h}$; 5 th: $0.0-0.6 \mathrm{~h}$; fig. $4 \mathrm{~d}$, e, respectively). Largely overlapping 95\% CI supported the consistency of each of these outcomes across the multiple icatibant-treated attacks (fig. $4 \mathrm{a}-\mathrm{e}$ ). Figure 5 shows the composite VAS scores and individual VAS symptom scores before treatment and at 1 and $4 \mathrm{~h}$ after treatment.

\section{Safety}

Icatibant was generally well tolerated in FAST-3; safety data for the controlled phase have been reported previously [8]. In the repeated-treatment population, $\geq 1 \mathrm{AE}$ was experienced by $39.8 \%$ (35/88), $35.7 \%$ (25/70), $36.4 \%$ (20/55), $21.6 \%(8 / 37)$ and $22.6 \%(7 / 31)$ of patients who had one, two, three, four or five icatibant-treated attacks, respectively. Frequently reported AEs ( $\geq 5 \%$ of patients in the first, second, third, fourth or fifth icatibant-treated attack groups) were a worsening or recurrence of HAE attacks $[8.0 \%(7 / 88), 4.3 \%(3 / 70), 12.7 \%$ (7/55), $2.7 \%$ $(1 / 37)$ and $0 \%$, respectively], headache $[8.0 \%(7 / 88), 4.3 \%$ (3/70), 3.6\% (2/55), $0 \%$ and $3.2 \%(1 / 31)$, respectively], and nasopharyngitis $[2.3 \%(2 / 88), 5.7 \%(4 / 70), 0 \%, 0 \%$ and $0 \%$, respectively]. Six severe AEs (headache, $\mathrm{n}=3$; dyspepsia, $\mathrm{n}=1$; noncardiac chest pain, $\mathrm{n}=1$; pain, $\mathrm{n}=$ 1) and two serious AEs (arrhythmia, $n=1$; noncardiac chest pain, $\mathrm{n}=1$ ) were considered related to icatibant treatment by the investigator. One of these drug-related serious AEs (arrhythmia) led to study discontinuation.

There were two notable serious AEs relating to laryngeal symptoms in the repeated-treatment population, both occurring in patients previously treated with icatibant but during subsequent attacks that were not treated with icatibant: 1 patient experienced laryngeal edema requiring upper airway management at 49 days after their most recent administration of icatibant for a previous attack, and another experienced a laryngeal HAE attack requiring treatment that included $\mathrm{C} 1$-inhibitor concentrate and trache-

Fig. 2. Kaplan-Meier curves showing median (95\% CI) times to onset of symptom relief (primary end point; a), onset of primary symptom relief (b), almost complete symptom relief (c), patient-assessed initial symptom improvement (d) and investigator-assessed initial symptom improvement (repeated-treatment population; e). ${ }^{1} \mathrm{~Pa}$ tients who did not achieve symptom relief within the observation period were censored at the last observation time. ${ }^{2}$ Patients who did not achieve symptom improvement within the observation period were censored at $48 \mathrm{~h}$. Note that the first icatibant-treated attack could have taken place in the controlled phase (only one injection permitted) or the OLE phase (up to three injections permitted).

(For figure see next page.)
48

Int Arch Allergy Immunol 2015;168:44-55 DOI: $10.1159 / 000441060$
Lumry/Farkas/Moldovan/Toubi/Baptista/ Craig/Riedl 

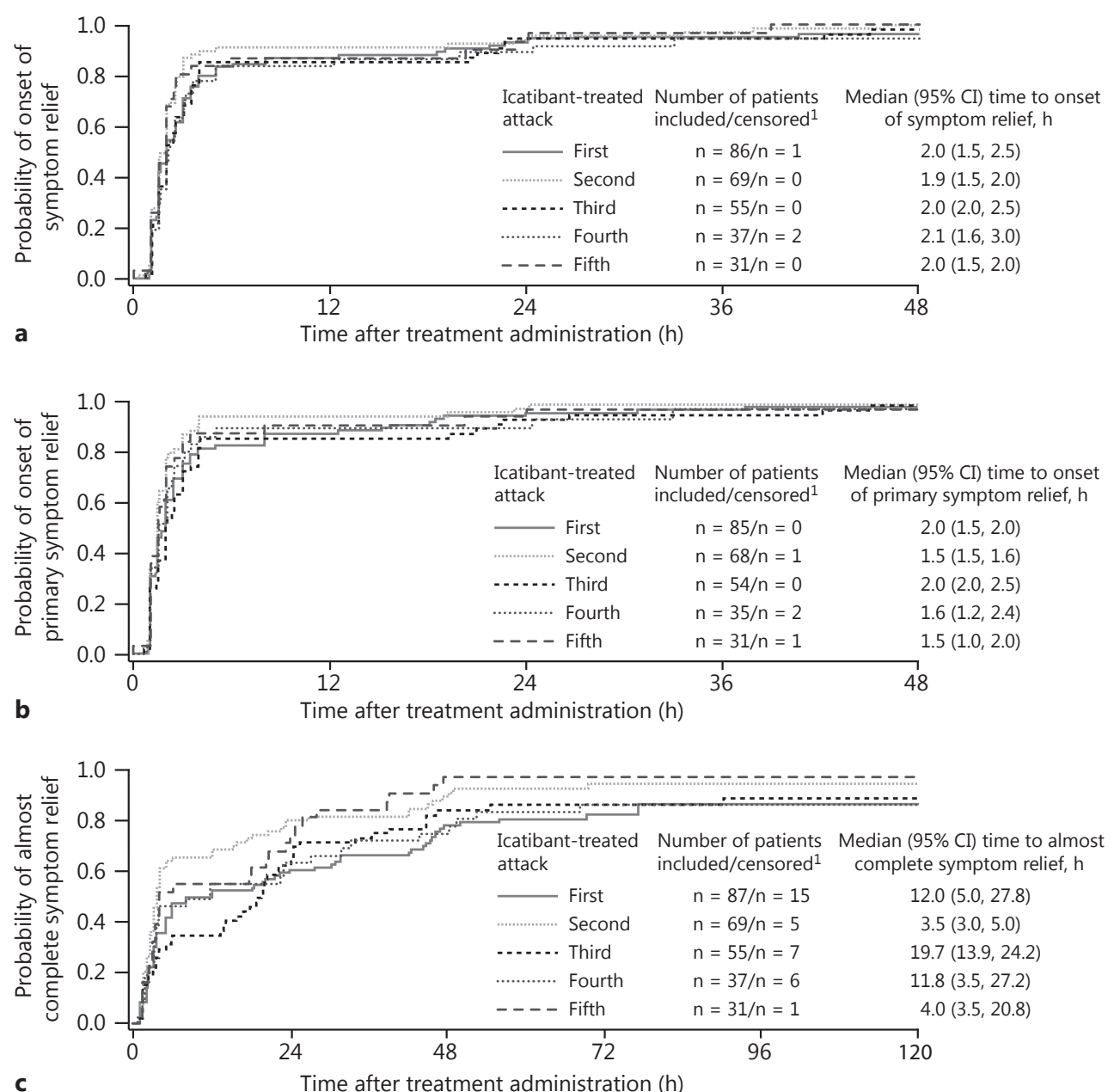

c Time after treatment administration (h)
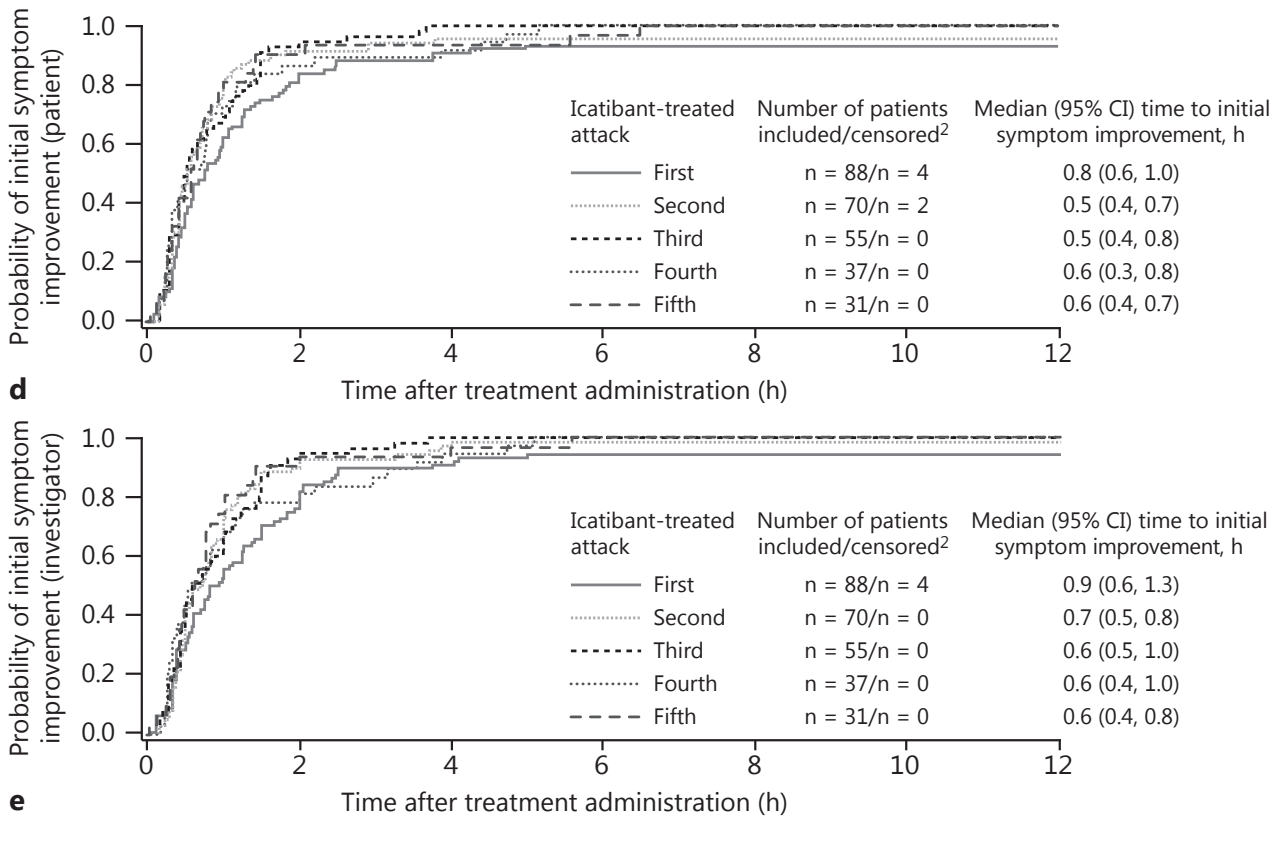

2 


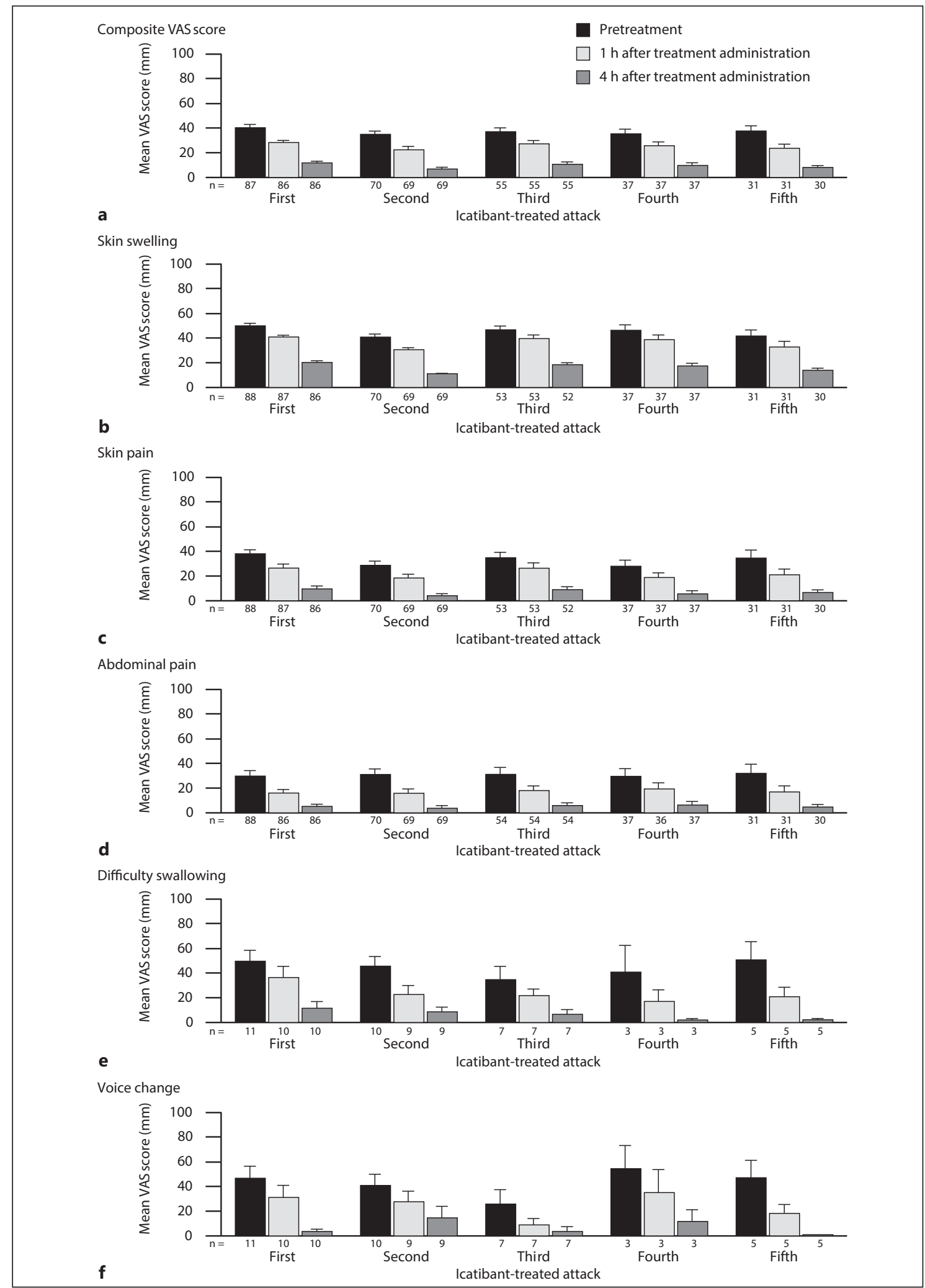

Fig. 3. Mean composite VAS scores (a) and individual VAS symptom scores (b-f) by number of icatibant-treated attacks at pretreatment and at 1 and $4 \mathrm{~h}$ after treatment (repeated-treatment population). Error bars indicate standard error. 

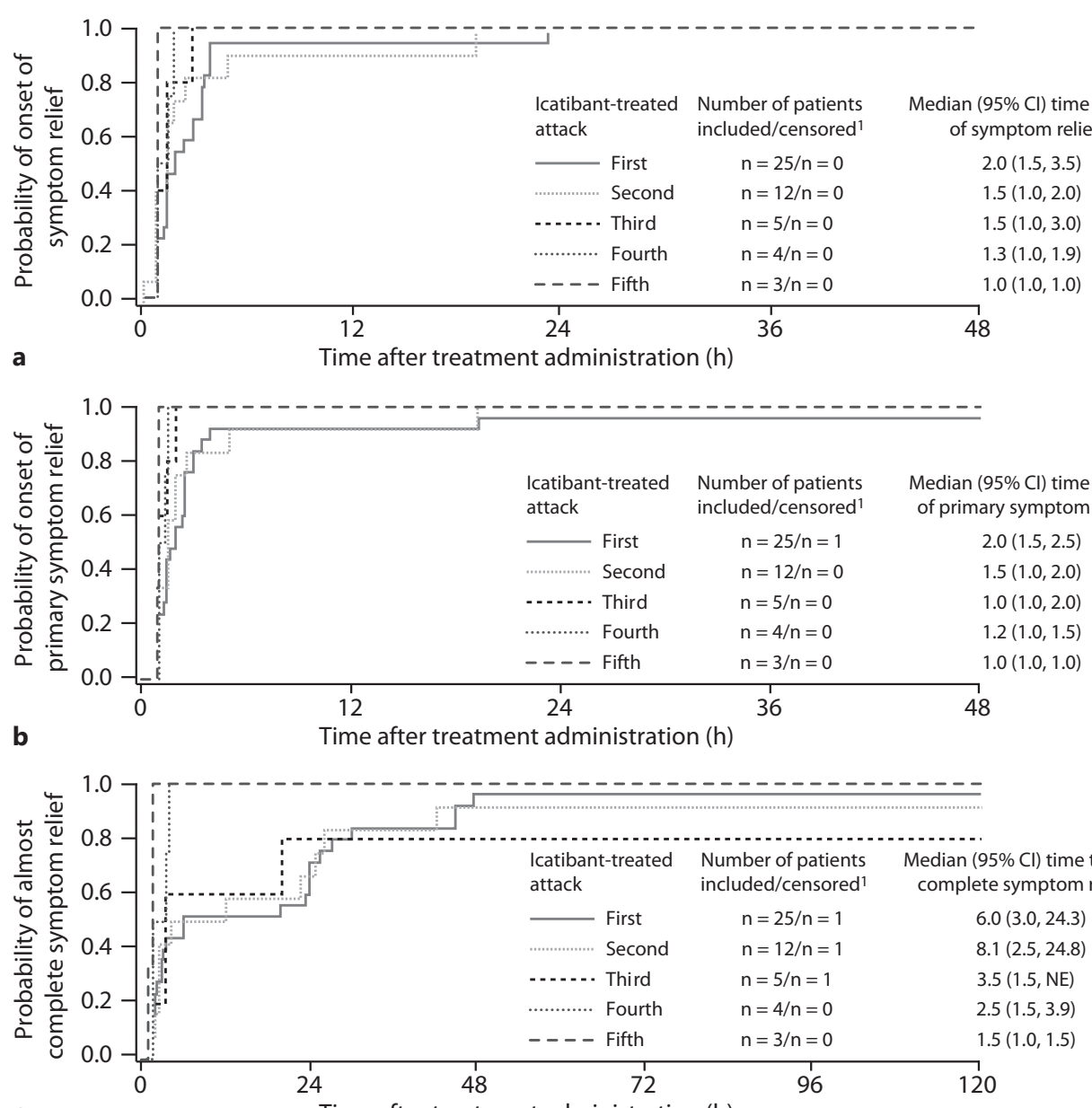

c Time after treatment administration (h)
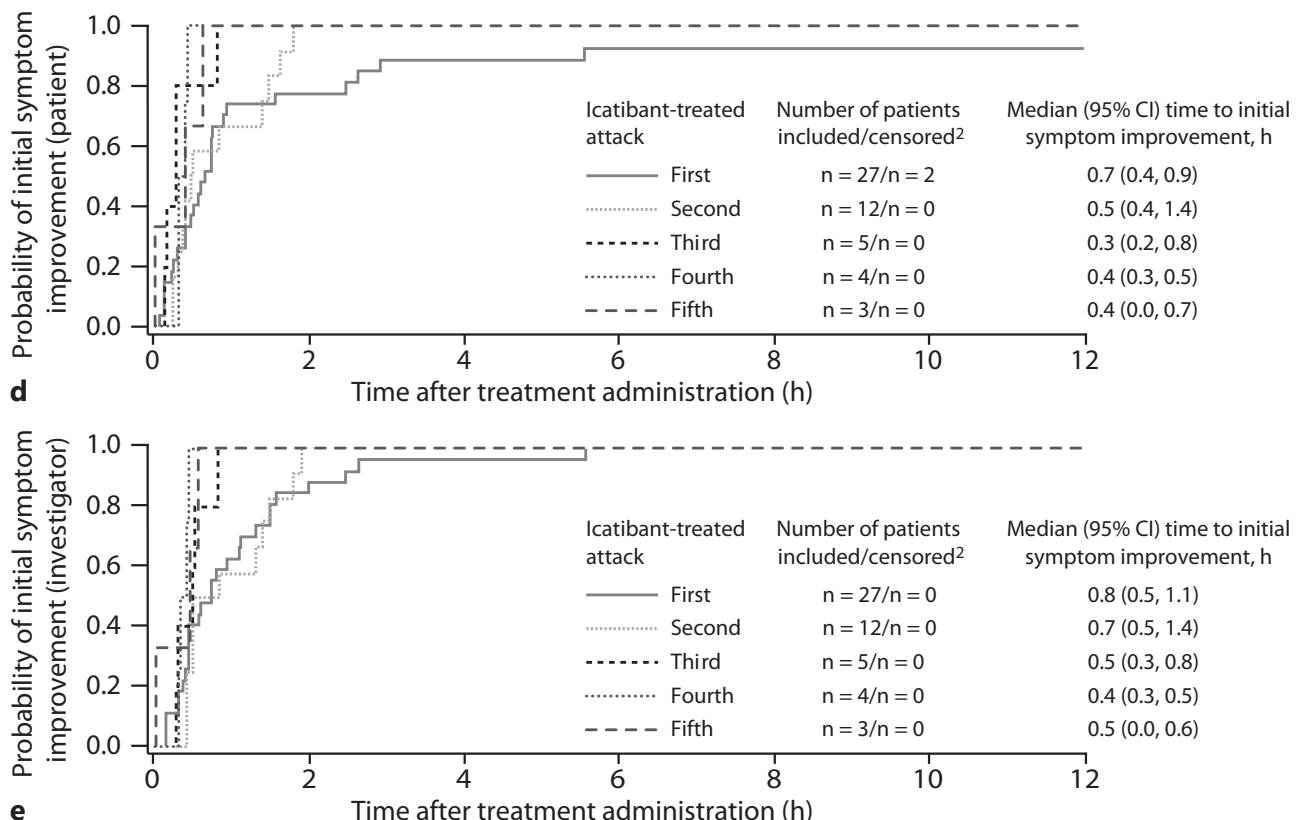

(For legend see next page.) 
otomy at 11 days after their most recent administration of icatibant for a previous attack. There was also one death in the repeated-treatment population: 1 patient who had received icatibant for six previous attacks died due to a laryngeal attack that occurred 139 days after the most recent dose of icatibant, administered during a previous attack.

Although injection-site reactions (documented separately from AEs) were reported in $94.6-98.2 \%$ of patients across the first, second, third, fourth or fifth icatibanttreated HAE attacks in the repeated-treatment population, most were mild to moderate in severity (8.1-19.4\% were rated as severe). The most frequently reported injection-site reactions were erythema (overall incidence, 89.2-98.2\%; severe, 5.4-19.4\%) and swelling (overall incidence, $75.7-86.4 \%$; severe, $1.4-8.1 \%$ ).

\section{Discussion}

Current treatment approaches for HAE rely on preventing or resolving recurring attacks $[11,12]$. As a result, patients have repeat exposure to their HAE-specific medications over the course of this chronic condition. Therefore, long-term efficacy and safety data for these drugs are of critical importance to explore any potential changes over time, such as the waning of efficacy or the emergence of new AEs, which could have implications for the management of patients in clinical practice.

With this in mind, these analyses of the controlled and OLE phases of the large, multicenter phase 3 FAST-3 study contribute to the evidence base for the sustained clinical profile of icatibant over time. Notably, icatibant consistently provided rapid onset of symptom relief across multiple HAE attacks (type I or II) at any location, as evaluated for up to five icatibant-treated attacks, using a range of patient- and investigator-reported end points. Indeed, both patient and investigator assessments indicated that initial symptom improvement was consistently

Fig. 4. Kaplan-Meier curves showing median (95\% CI) times to onset of symptom relief (primary end point; a), onset of primary symptom relief (b), almost complete symptom relief (c), patientassessed initial symptom improvement (d) and investigator-assessed initial symptom improvement (laryngeal repeated-treatment population; e). NE $=$ Not estimable. ${ }^{1}$ Patients who did not achieve symptom relief within the observation period were censored at the last observation time; postbaseline composite VAS scores were only available for $25 / 27$ patients with icatibant-treated laryngeal attacks. ${ }^{2}$ Patients who did not achieve symptom improvement within the observation period were censored at $48 \mathrm{~h}$. achieved within a median of $0.5-0.9 \mathrm{~h}$. In addition, treatment was generally well tolerated. These findings warrant attention from the clinical community, particularly considering the unpredictable nature of HAE and its widely recognized association with a reduced quality of life, depressive symptomatology and reduced work productivity $[13,14]$. Although specific data are currently lacking, it is expected that access to effective treatments will reduce morbidity and the burden of this condition.

Given the potential for fatal asphyxiation [2, 3], patients with laryngeal edema are a particularly important subpopulation for study in order to establish the efficacy of treatment in resolving these life-threatening symptoms. Accordingly, these patients were specifically assessed in a post hoc analysis, which indicated that icatibant was associated with sustained rapid symptom relief across multiple laryngeal attacks. Although 2 patients experienced serious laryngeal AEs that required airway management, both occurred during subsequent attacks that were not treated with icatibant. These observations substantiate the potential of icatibant in reducing the serious outcomes associated with laryngeal HAE attacks.

In this analysis, times to onset of symptom relief and almost complete symptom relief for first to fifth HAE attacks at any location were consistent with data obtained with icatibant in the FAST- 1 and FAST- 2 studies. The median times to onset of primary symptom relief and almost complete symptom relief were $1.0-2.0 \mathrm{~h}$ and $4.8-$ $55.0 \mathrm{~h}$, respectively, for the first to tenth cutaneous and/ or abdominal attacks in the OLE phase of FAST-1, and 1.3-3.9 $\mathrm{h}$ and $6.5-33.1 \mathrm{~h}$ for the first to fifth cutaneous and/or abdominal attacks in the controlled and OLE phases of FAST-2 $[9,10]$. Similarly, consistency of response over the repeated treatment of multiple attacks has also been reported with other HAE medications, including the kallikrein inhibitor ecallantide [15] and C1esterase inhibitors $[16,17]$. However, meaningful comparisons of specific outcomes with different medications are precluded by variations in study designs and efficacy assessments and a lack of head-to-head studies.

Although second or third injections of icatibant were permitted as needed in the OLE phase of FAST-3, the majority of HAE attacks were successfully treated with a single injection only. Again, this observation is consistent with the findings of the FAST-1 and FAST-2 OLE phases $[9,10]$ and studies of other HAE medications $[18,19]$.

In addition to efficacy data, the analyses also indicated that icatibant is generally well tolerated with repeated use, and safety data were consistent with the controlled phases of the phase 3 studies of icatibant $[7,8]$. Of particular
Lumry/Farkas/Moldovan/Toubi/Baptista/ Craig/Riedl 


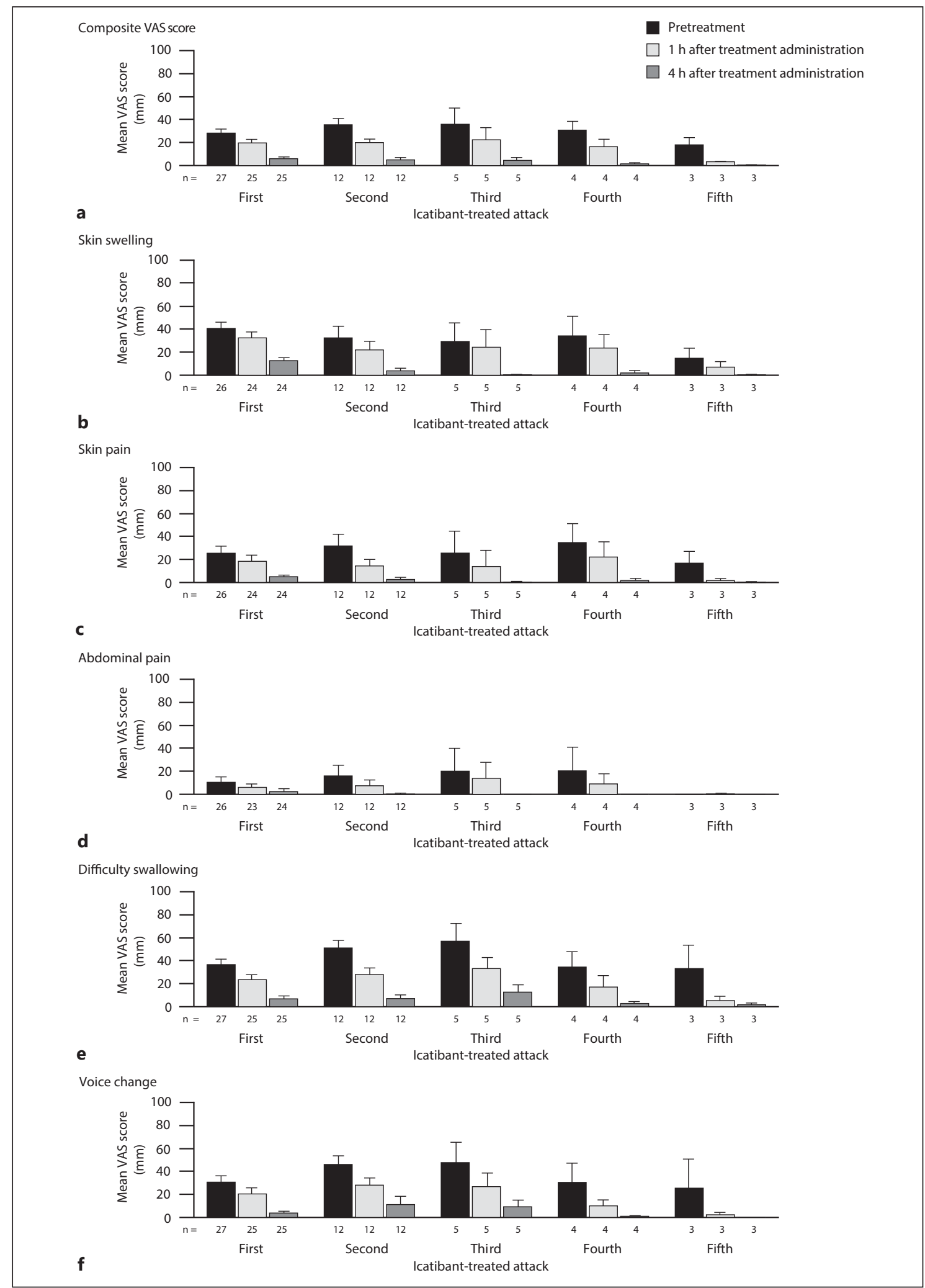

Fig. 5. Mean composite VAS symptom scores (a) and individual VAS symptom scores (b-f) at pretreatment and 1 and $4 \mathrm{~h}$ after treatment with icatibant (laryngeal repeated-treatment population). Error bars indicate standard error. 
note, while frequent, the majority of injection-site reactions were mild to moderate in severity and resolved, as was the case in the controlled phase of FAST-3 (see Lumry et al. [8] for further details); this may be encouraging for patients who are considering self-administration of icatibant at home. Self-administration is recommended where possible to allow prompt treatment of HAE attacks $[11,12,20-23]$ and has been associated with improved outcomes [24]. The EASSI study [25] demonstrated that with appropriate training, patients were able to identify the start of an HAE attack and determine when to selfadminister icatibant. In this study, self-administration was associated with a high degree of patient satisfaction, as well as convenience and ease of use. Home treatment with icatibant administered by a health care professional has also been described as an option, with outcomes similar to those reported in clinical settings [26].

The potential limitations of this analysis include the combination of data from two phases of the FAST-3 study with slightly different designs. However, the pooling of icatibant-treated attacks across the controlled and OLE phases permitted a broader data set for the assessment of the efficacy and safety of repeated treatment with icatibant in this study. Of note, only a single injection of icatibant was permitted in the controlled phase, whereas up to three injections per attack could be administered in the OLE phase. In addition, the open-label design of the extension phase of FAST-3 may have led patients to have some expectation of benefit. However, the consistency of results, both across multiple attacks and when compared to the previously reported data in the controlled phase only, using both patient- and investigator-assessed end points, suggests that knowledge of treatment assignment had a minimal impact on the study results.

In summary, the analyses presented here support existing evidence that the efficacy and safety profiles of icatibant are sustained when used to treat multiple cutaneous, abdominal or laryngeal HAE attacks type I or II.

\section{Acknowledgments}

The work was conducted in 67 different centers in 11 countries. The FAST-3 investigators included: Australia: Cook M, Katelaris C, Smith W; Canada: Ritchie B; Hungary: Farkas H; Israel: Kivity S, Toubi E; Mexico: Gonzalez-Diaz S; Romania: Moldovan D; Russia: Emelyanov A, Goryachkina L, Klimko N, Sidorenko I; South Africa: Potter PC; Turkey: Buyukozturk S, Karakaya G; Ukraine: Kaydashev I, Neyko Y; USA: Ajmani A, Bahna S, Baker J, Banerji A, Bernstein JA, Bonner J, Busse P, Craig T, Davis-Lorton M, Diaz JD, Frank M, Gleich G, Grant JA, Hogan MB, Hurewitz D, Huston D, Levy RJ, Li HH, Lockey R, Lumry W, McNeil D, Moy JN, Nadeau K, Offenberger J, Riedl M, Schenkel E, Sitz KV, Soteres D, Wedner HJ, Yang W, Zuraw B.

The FAST-3 study was funded by Shire, Lexington, Mass., USA. Editorial support was provided by Hannah FitzGibbon of Complete Medical Communications, with funding from Shire, Eysins, Switzerland.

\section{References}

1 Bork K, Staubach P, Eckardt AJ, Hardt J: Symptoms, course, and complications of abdominal attacks in hereditary angioedema due to $\mathrm{C} 1$ inhibitor deficiency. Am J Gastroenterol 2006;101:619-627.

2 Bork K, Siedlecki K, Bosch S, Schopf RE, Kreuz W: Asphyxiation by laryngeal edema in patients with hereditary angioedema. Mayo Clin Proc 2000;75:349-354.

3 Bork K, Hardt J, Witzke G: Fatal laryngeal attacks and mortality in hereditary angioedema due to C1-INH deficiency. J Allergy Clin Immunol 2012;130:692-697.

4 Cicardi M, Bergamaschini L, Cugno M, Beretta A, Zingale LC, Colombo M, Agostoni A: Pathogenetic and clinical aspects of $\mathrm{C} 1$ inhibitor deficiency. Immunobiology 1998;199: 366-376.

5 Cugno M, Nussberger J, Cicardi M, Agostoni A: Bradykinin and the pathophysiology of angioedema. Int Immunopharmacol 2003;3: 311-317.
6 Bork K: Diagnosis and treatment of hereditary angioedema with normal C1 inhibitor. Allergy Asthma Clin Immunol 2010;6:15.

7 Cicardi M, Banerji A, Bracho F, et al: Icatibant, a new bradykinin-receptor antagonist, in hereditary angioedema. N Engl J Med 2010; 363:532-541.

8 Lumry WR, Li HH, Levy RJ, Potter PC, Farkas $\mathrm{H}$, Moldovan D, Riedl M, Li H, Craig T, Bloom BJ, Reshef A: Randomized placebocontrolled trial of the bradykinin $\mathrm{B}_{2}$ receptor antagonist icatibant for the treatment of acute attacks of hereditary angioedema: the FAST-3 trial. Ann Allergy Asthma Immunol 2011; 107:529-537.

9 Malbrán A, Riedl M, Ritchie B, Smith WB, Yang W, Banerji A, Hébert J, Gleich GJ, Hurewitz D, Jacobson KW, Bernstein JA, Khan DA, Kirkpatrick H, Resnick D, Li H, Fernández Romero S, Lumry W: Repeat treatment of acute hereditary angioedema attacks with open-label icatibant in the FAST-1 trial. Clin Exp Immunol 2014;177:544-553.
10 Bas M, Greve J, Hoffmann TK, Reshef A, Aberer W, Maurer M, Kivity S, Farkas H, Floccard B, Arcoleo F, Martin L, Sitkauskiene B, Bouillet L, Schmid-Grendelmeier P, Li H, Zanichelli A: Repeat treatment with icatibant for multiple hereditary angioedema attacks: FAST-2 open-label study. Allergy 2013;68:1452-1459.

11 Cicardi M, Bork K, Caballero T, Craig T, Li $\mathrm{HH}$, Longhurst $\mathrm{H}$, Reshef A, Zuraw B; HAWK (Hereditary Angioedema International Working Group): Evidence-based recommendations for the therapeutic management of angioedema owing to hereditary $\mathrm{C} 1$ inhibitor deficiency: consensus report of an International Working Group. Allergy 2012;67:147-157.

12 Craig T, Aygören-Pürsün E, Bork K, Bowen T, Boysen H, Farkas H, Grumach A, Katelaris $\mathrm{CH}$, Lockey R, Longhurst H, Lumry W, Magerl M, Martinez-Saguer I, Ritchie B, Nast A, Pawankar R, Zuraw B, Maurer M: WAO guideline for the management of hereditary angioedema. World Allergy Organ J 2012;5: 182-199. 
13 Banerji A: The burden of illness in patients with hereditary angioedema. Ann Allergy Asthma Immunol 2013;111:329-336.

14 Lumry WR, Castaldo AJ, Vernon MK, Blaustein $\mathrm{MB}$, Wilson DA, Horn PT: The humanistic burden of hereditary angioedema: impact on health-related quality of life, productivity, and depression. Allergy Asthma Proc 2010;31:407-414.

15 Lumry WR, Bernstein JA, Li HH, MacGinnitie AJ, Riedl M, Soteres DF, Craig TJ, Campion M, Iarrobino R, Stolz LE, Pullman WE: Efficacy and safety of ecallantide in treatment of recurrent attacks of hereditary angioedema: open-label continuation study. Allergy Asthma Proc 2013;34:155-161.

16 Craig TJ, Bewtra AK, Hurewitz D, Levy R, Janss G, Jacobson KW, Packer F, Bernstein JA, Rojavin MA, Machnig T, Keinecke HO, Wasserman RL: Treatment response after repeated administration of $\mathrm{C} 1$ esterase inhibitor for successive acute hereditary angioedema attacks. Allergy Asthma Proc 2012;33:354361.

17 Riedl MA, Levy RJ, Suez D, Lockey RF, Baker JW, Relan A, Zuraw BL: Efficacy and safety of recombinant $\mathrm{C} 1$ inhibitor for the treatment of hereditary angioedema attacks: a North American open-label study. Ann Allergy Asthma Immunol 2013;110:295-299.
$18 \mathrm{Li} \mathrm{HH}$, Campion M, Craig TJ, Soteres DF, Riedl M, Lumry WR, MacGinnitie AJ, Shea EP, Bernstein JA: Analysis of hereditary angioedema attacks requiring a second dose of ecallantide. Ann Allergy Asthma Immunol 2013; 110:168-172.

19 Craig TJ, Bewtra AK, Bahna SL, Hurewitz D, Schneider LC, Levy RJ, Moy JN, Offenberger J, Jacobson KW, Yang WH, Eidelman F, Janss G, Packer FR, Rojavin MA, Machnig T, Keinecke HO, Wasserman RL: C1 esterase inhibitor concentrate in 1085 Hereditary Angioedema attacks - final results of the I.M.P.A.C.T.2 study. Allergy 2011;66:1604-1611.

20 Bowen T, Cicardi M, Farkas H, et al: 2010 International consensus algorithm for the diagnosis, therapy and management of hereditary angioedema. Allergy Asthma Clin Immunol 2010;6:24.

21 Longhurst HJ, Farkas H, Craig T, AygörenPürsün E, Bethune C, Bjorkander J, Bork K, Bouillet L, Boysen H, Bygum A, Caballero T, Cicardi M, Dempster J, Gompels M, Gooi J, Grigoriadou S, Huffer U, Kreuz W, Levi MM, Long J, Martinez-Saguer I, Raguet M, Reshef A, Bowen T, Zuraw B: HAE international home therapy consensus document. Allergy Asthma Clin Immunol 2010;6:22.

22 Zuraw B, Banerji A, Bernstein JA, Busse PJ, Christiansen SC, Davis-Lorton M, Frank MM, Li HH, Lumry WR, Riedl M: US Hereditary Angioedema Association Medical Advisory Board 2013 recommendations for the management of hereditary angioedema due to $\mathrm{C} 1$ inhibitor deficiency. J Allergy Clin Immunol Pract 2013;1:458-467.
23 Zuraw BL, Bernstein JA, Lang DM, Craig T, Dreyfus D, Hsieh F, Khan D, Sheikh J, Weldon D, Bernstein DI, Blessing-Moore J, Cox L, Nicklas RA, Oppenheimer J, Portnoy JM, Randolph CR, Schuller DE, Spector SL, Tilles SA, Wallace D; American Academy of Allergy, Asthma and Immunology; American College of Allergy, Asthma and Immunology: A focused parameter update: hereditary angioedema, acquired $\mathrm{C} 1$ inhibitor deficiency, and angiotensin-converting enzyme inhibitor-associated angioedema. J Allergy Clin Immunol 2013;131:1491-1493

24 Maurer M, Aberer W, Bouillet L, Caballero T, Fabien V, Kanny G, Kaplan A, Longhurst H, Zanichelli A; IOS Investigators: Hereditary angioedema attacks resolve faster and are shorter after early icatibant treatment. PLoS One 2013;8:e53773.

25 Aberer W, Maurer M, Reshef A, Longhurst H, Kivity S, Bygum A, Caballero T, Bloom B, Nair N, Malbrán A: Open-label, multicenter study of self-administered icatibant for attacks of hereditary angioedema. Allergy 2014; 69:305-314.

26 Farkas H, Csuka D, Zotter Z, Varga L, Böröcz Z, Temesszentandrási G, Jakab L, Karádi I: Home treatment of hereditary angioedema with icatibant administered by health care professionals. J Allergy Clin Immunol 2012; 129:851-852. 